\title{
A Fractional-Order Model for the Spread of Pests in Tea Plants
}

\author{
Moustafa El-Shahed ${ }^{1}$, A. M. Ahmed ${ }^{2}$, Ibrahim. M. E. Abdelstar ${ }^{2,3}$ \\ ${ }^{1}$ Department of Mathematics, Faculty of Arts and Sciences, Qassim University, P.O. Box 3771, Qassim, Unizah \\ 51911, Saudi Arabia. \\ ${ }^{2}$ Department of Mathematics, Faculty of Science, Al-Azhar University, Nasr City, P.O. Box: 11884, Cairo, Egypt. \\ ${ }^{3}$ Quantitative Methods Unit, Faculty of Business and Economics, Qassim University, P.O. Box 6633, Qassim, \\ Buraidah 51452, Saudi Arabia. \\ Email: elshahedm@yahoo.com, ahmedelkb@yahoo.com, and essonny19@gmail.com
}

\begin{abstract}
In this paper, a fractional-order model for the spread of pests in tea plants is presented. This model consists of three components: tea plant, pest, and predator. The stability of the boundary and positive fixed points is studied. The global stability properties of the positive equilibrium point are also investigated. In addition, fractional Hopf bifurcation conditions for the model are proposed. The generalized Adams-Bashforth-Moulton method is used to solve and simulate the system of fractional differential equations.
\end{abstract}

Keywords: Natural enemy; fractional order; stability; numerical method.

\section{Introduction}

Camellia sinensis is a tree or small shrub that belongs to the family Theaceae. It is grown for its leaves, which are used to make tea, an aromatic beverage. This alternate-branching plant produces matte-green elliptical leaves with a leathery texture and a serrate margin. Although the tea plant can grow to become a tree with a bowl-shaped canopy, it is generally smaller and shrub-like as a consequence of cultivation and pruning. Flowering is prevented in tea plants meant for tea cultivation. When they are allowed to flower, tea plants produce fragrant white flowers singly or in small clusters. Tea trees can grow to $15 \mathrm{~m}$ ( $49 \mathrm{ft})$ in height in the wild and can have a life expectancy of 30 to 50 years. [15, 31].

Because of their potential applications in science and engineering, interest in fractional calculus and fractional differential equations has grown dramatically in recent decades $[9,19,28]$. In this paper, we consider the fractional-order model for a tritrophic model consisting of tea plant, pest, and predator. We give a detailed analysis of the asymptotic and global stability of the model. To solve and simulate the system of fractional differential equations, we use the Adams-Bashforth-Moulton algorithm.

\section{Model Formulation}

The tritrophic food chain model for usefulness of biocontrol of pests in tea can be written as a set of three coupled nonlinear ordinary differential equations as follows [22]:

$$
\begin{aligned}
& \frac{\mathrm{d} X}{\mathrm{~d} T}=r X\left(1-\frac{X}{K}\right)-\frac{b X Y}{a+X} \\
& \frac{\mathrm{d} X}{\mathrm{~d} T}=-\mathrm{d} Y+\frac{m X Y}{a+X}-p Y Z \\
& \frac{\mathrm{d} Z}{\mathrm{~d} T}=-\mu Z+q Y Z
\end{aligned}
$$

The above model has three populations. The host (tea plant) whose population density at time $t$ is denoted by $X$, the pest whose population density is denoted by $Y$ and the predator whose population density is denoted by $Z$.ll denotes the death rate of the pests, $m$ is the conversion rate of the pests, $p$ is the quantity that represents the decrease in the growth rate of the pests due to predator attack, $q$ is the rate of increase of predator population, $K$ is the carrying capacity, $r$ denotes the intrinsic growth 
rate constant and $\mu$ denotes the intrinsic mortality rate of the predators. Here all the parameters $r, K, b, a, d, m, q$ are positive [22].

Fractional order models are more accurate than integer-order models as fractional order models allow more degrees of freedom. Fractional differential equations also serve as an excellent tool for the description of hereditary properties of various materials and processes. The presence of memory term in such models not only takes into account the history of the process involved but also carries its impact to present and future development of the process. Fractional differential equations are also regarded as an alternative model to nonlinear differential equations. In consequence, the subject of fractional differential equations is gaining much importance and attention. For some recent work on fractional differential equations, see $[19,28]$. Now we introduce fractional order into the ODE model by Maiti et al [22]. The new system is described by the following set of fractional order differential equations:

$$
\begin{aligned}
& D_{t}^{\alpha} X=r X\left(1-\frac{X}{K}\right)-\frac{b X Y}{a+X} \\
& D_{t}^{\alpha} Y=-d Y+\frac{m X Y}{a+X}-p Y Z \\
& D_{t}^{\alpha} Z=-\mu Z+q Y Z
\end{aligned}
$$

where $D_{t}^{\alpha}$ is the Caputo fractional derivative. Because model (2.2) monitors the dynamics of human populations, all the parameters are assumed to be non-negative. Furthermore, it can be shown that all state variables of the model are non-negative for all time $t \geq 0$ (see, for instance, [5]).

Lemma 2.1. The solutions of the system (2.2) exist in $\mathbb{R}_{+}^{3}$ and uniformly bounded. proof. Let $(X(t), Y(t), Z(t))$ be any solution of the system $(2.2)$ with positive initial conditions. Since

$$
D_{t}^{\alpha} X \leq r X\left(1-\frac{X}{K}\right)
$$

by Lemma 9 of [20] we have

$$
X(t) \leq X(0) E_{\alpha}\left(r t^{\alpha}\right)
$$

where $E_{\alpha}$ is the Mittag-Leffler function. Let

$$
W=\frac{m}{b} X+Y+\frac{p}{q} Z
$$

then

$$
\begin{aligned}
D_{t}^{\alpha} W & =\frac{m}{b} D_{t}^{\alpha} X+D_{t}^{\alpha} Y+\frac{p}{q} D_{t}^{\alpha} Z \\
& =\frac{r m}{b} X\left(1-\frac{X}{K}\right)-d Y-\frac{p \mu}{q} Z \\
& =\frac{r m}{b} X-\frac{r m}{b K} X^{2}-d Y-\frac{p \mu}{q} Z \\
& =\frac{2 r m}{b} X-\frac{r m}{b K} X^{2}-\frac{r m}{b} X-d Y-\frac{p \mu}{q} Z \\
& \leq \frac{2 r m}{b} X-k W
\end{aligned}
$$

where $k=\min \{r, d, \mu\}$. By Lemma 9 of [20] again, we have

$$
0 \leq W(X, Y, Z) \leq W(X(0), Y(0), Z(0)) E_{\alpha}\left(-k t^{\alpha}\right)+\frac{2 r m}{b} E_{\alpha, \alpha+1}\left(-k t^{\alpha}\right)=W_{1}
$$

where $E_{\alpha}$ is the Mittag-Leffler function. Therefore, all solutions of the model (2.2) with initial conditions in $\Omega$ s.t.

$$
\Omega=\left\{(X, Y, Z) \in W: 0 \leq W \leq W_{1}\right\}
$$

remain in $\Omega$ for all $t>0$. Thus, region $\Omega$ is positively invariant with respect to model $(2.2)$. 
In the following, we will study the dynamics of system (2.2).

\section{Equilibrium Point and Stability}

In the following, we discuss the stability of the commensurate fractional ordered dynamical system:

$$
D_{t}^{\alpha} x_{i}=f_{i}\left(x_{1}, x_{2}, x_{3}\right), \quad \alpha \in(0,1), \quad 1 \leq i \leq 3
$$

Let $E=\left(x_{1}^{*}, x_{2}^{*}, x_{3}^{*}\right)$ be an equilibrium point of system (3.1) and $x_{i}=x_{i}^{*}+\eta_{i}$ where $\eta_{i}$ is a small disturbance from a fixed point. Then

$$
\begin{aligned}
D_{t}^{\alpha} \eta_{i} & =D_{t}^{\alpha} x_{i} \\
& =f_{i}\left(x_{1}^{*}+\eta_{1}, x_{2}^{*}+\eta_{2}, x_{3}^{*}+\eta_{3}\right) \\
& \approx \eta_{1} \frac{\partial f_{i}(E)}{\partial x_{1}}+\eta_{2} \frac{\partial f_{i}(E)}{\partial x_{2}}+\eta_{3} \frac{\partial f_{i}(E)}{\partial x_{3}}
\end{aligned}
$$

System (3.2) can be written as:

$$
D_{t}^{\alpha} \eta=J \eta
$$

where $\eta=\left(\eta_{1}, \eta_{2}, \eta_{3}\right)^{T}$ and $J$ is the Jacobian matrix evaluated at the equilibrium points. Using Matignon's results [23], it follows that the linear autonomous system (3.3) is asymptotically stable if $|\arg (\lambda)|>\frac{\alpha \pi}{2}$ is satisfied for all eigenvalues of matrix $J$ at the equilibrium point $E=\left(x_{1}^{*}, x_{2}^{*}, x_{3}^{*}\right)$. If $\phi(x)=x^{3}+a_{1} x^{2}+a_{2} x+a_{3}$, let $D(\phi)$ denote the discriminant of a polynomial $\phi$, then

$$
D(\phi)=-\left|\begin{array}{ccccc}
1 & a_{1} & a_{2} & a_{3} & 0 \\
0 & 1 & a_{1} & a_{2} & a_{3} \\
3 & 2 a_{1} & a_{2} & 0 & 0 \\
0 & 3 & 2 a_{1} & a_{2} & 0 \\
0 & 0 & 3 & 2 a_{1} & a_{2}
\end{array}\right|=18 a_{1} a_{2} a_{3}+\left(a_{1} a_{2}\right)^{2}-4 a_{3} a_{1}^{3}-4 a_{2}^{3}-27 a_{3}^{2}
$$

Following $[1,2,3,23]$, we have the proposition.

Proposition 3.1. One assumes that $E$ exists in $R_{+}^{3}$.

1. If the discriminant of $\phi(x), D(\phi)$ is positive and Routh-Hurwitz are satisfied, that is, $D(\phi)>0$, $a_{1}>0, a_{3}>0, a_{1} a_{2}>a_{3}$, then $E$ is locally asymptotically stable.

2. If $D(\phi)<0, a_{1}>0, a_{2}>0, a_{1} a_{2}=a_{3}, \alpha \in[0,1)$, then $E$ is locally asymptotically stable.

3. If $D(\phi)<0, a_{1}<0, a_{2}<0, \alpha>\frac{2}{3}$, then $E$ is unstable.

4. The necessary condition for the equilibrium point $E$, to be locally asymptotically stable, is $a_{3}>0$.

To evaluate the equilibrium points, let

$$
D_{t}^{\alpha} X=0, D_{t}^{\alpha} Y=0, D_{t}^{\alpha} Z=0
$$

Then:

1. The first trivial equilibrium point is $E_{0}=(0,0,0)$. The point $E_{0}$ always exists.

The Jacobian matrix $J\left(E_{0}\right)$ for the system given in (2.2), evaluated at $E_{0}$, is as follows:

$$
J\left(E_{0}\right)=\left(\begin{array}{ccc}
r & 0 & 0 \\
0 & -d & 0 \\
0 & 0 & -\mu
\end{array}\right)
$$


Theorem 3.2. The trivial equilibrium point $E_{0}$ of system (2.2) is a saddle point.

proof. The trivial equilibrium point $E_{0}$ is locally asymptotically stable if all the eigenvalues $\lambda_{0 i}$, $i=1,2,3$ of $J\left(E_{0}\right)$ satisfy Matignon's conditions. The eigenvalues corresponding to the equilibrium $E_{0}$ are $\lambda_{01}=r, \lambda_{02}=-d$ and $\lambda_{03}=-\mu$.

Then we have $\lambda_{01}>0, \lambda_{02}<0$ and $\lambda_{03}<0$. It follows that the node equilibrium point of system (2.2) is a saddle point, with nonempty stable manifolds and an unstable manifold.

2. The second equilibrium point is $E_{1}=(K, 0,0)$ when the pests are absent from the tea, in this case $(Y=Z=0)$; therefore, the tea is fully susceptible. The point $E_{1}$ always exists.

Theorem 3.3. For the system (2.2), the basic reproduction number is

$$
R_{0}=\frac{m K}{d(a+K)}
$$

proof. We use the next generation method to find the basic reproduction number for the system (2.2). When we rewrite the equations by which classes of the pest population $Y$ and the predator population $Z$ first and then the rest of the equations, we have the following:

$$
\begin{aligned}
& D_{t}^{\alpha} Y=-d Y+\frac{m X Y}{a+X}-p Y Z \\
& D_{t}^{\alpha} Z=-\mu Z+q Y Z \\
& D_{t}^{\alpha} X=r X\left(1-\frac{X}{K}\right)-\frac{b X Y}{a+X}
\end{aligned}
$$

We make matrices $f, v$, such that the system (3.4) has the form

$$
\frac{d x}{d t}=f(x)-v(x)
$$

where

$$
f(x)=\left[\begin{array}{c}
f_{1} \\
f_{2} \\
f_{3}
\end{array}\right]=\left[\begin{array}{c}
\frac{m X Y}{a+X} \\
q Y Z \\
0
\end{array}\right], v(x)=\left[\begin{array}{l}
v_{1} \\
v_{2} \\
v_{3}
\end{array}\right]=\left[\begin{array}{c}
d Y+p Y Z \\
\mu Z \\
\frac{b X Y}{a+X}-r X\left(1-\frac{X}{K}\right)
\end{array}\right]
$$

We make matrices $F, V$ such that

and then

$$
F(x)=\left[\begin{array}{ll}
\frac{\partial f_{1}}{\partial Y} & \frac{\partial f_{1}}{\partial \mathrm{Z}} \\
\frac{\partial f_{2}}{\partial Y} & \frac{\partial f_{2}}{\partial \mathrm{Z}}
\end{array}\right], \quad V(x)=\left[\begin{array}{ll}
\frac{\partial v_{1}}{\partial Y} & \frac{1}{\partial v} \\
\frac{\partial v_{2}}{\partial Y} & \frac{\partial v_{2}}{\partial \mathrm{Z}}
\end{array}\right]
$$

$$
F(x)=\left[\begin{array}{cc}
\frac{m X}{a+X} & 0 \\
q Z & q Y
\end{array}\right], \quad V(x)=\left[\begin{array}{cc}
d+p Z & p Y \\
0 & \mu
\end{array}\right]
$$

at the equilibrium point $E_{1}=(K, 0,0)$, to get the eigenvalues of $F \cdot V^{-1}$ one solves the equation

$$
\left|F \cdot V^{-1}-\lambda I\right|=0
$$

where $\lambda$ is the eigenvalues and $I$ is the identity matrix. Then $\lambda_{1}=\frac{m K}{d(a+K)}, \lambda_{2}=0$. It then follows that the spectral radius of the matrix $F \cdot V^{-1}$ is $\rho\left(F \cdot V^{-1}\right)=\max \left(\lambda_{i}\right), i=1,2$. Then $R_{0}=\frac{m K}{d(a+K)}$. $\square$ 
3. By (2.2), the third point is $E_{2}=\left(\frac{a d}{m-d}, \frac{\operatorname{ramd}(a+K)\left(R_{0}-1\right)}{K b(m-d)^{2}}, 0\right)$, which is free of the predator.

4. The fourth point is $E_{3}=\left(X_{3}, Y_{3}, Z_{3}\right)$ where

$$
\begin{aligned}
& X_{3}=\frac{1}{2}(R+K-a) \\
& Y_{3}=\frac{\mu}{q} \\
& Z_{3}=\frac{(R+K)(m-d)-a(m+d)}{p(R+K+a)} \\
& R=\sqrt{(K+a)^{2}-\frac{4 K b \mu}{r q}}
\end{aligned}
$$

\section{Remark 1.}

1) The free pest, whose population density is denoted by $Y$ does not exist. The predator $Z$, being a natural enemy of the concerned pest, preys on $Y$ and only on $Y$. So if $Y=0$, then it should be that $Z=0$ is the free equilibrium point $E_{1}$ again.

2) $E_{2}$ must have a nonnegative component, then the following condition exists: $m>d$ and $R_{0}>1$.

3) $E_{3}$ must have a nonnegative component, then the following condition exists: $\operatorname{arq}>b \mu$, $(R+K)(m-d)>a(m+d)$.

The Jacobian matrix $J\left(E_{1}\right)$ for the system given in (2.2), evaluated at $E_{1}$, is as follows:

$$
J\left(E_{1}\right)=\left[\begin{array}{ccc}
-r & \frac{b K}{a+K} & 0 \\
0 & d\left(R_{0}-1\right) & 0 \\
0 & 0 & -\mu
\end{array}\right]
$$

Theorem 3.4. The equilibrium point $E_{1}$ is unstable.

Proof. The equilibrium point $E_{1}$ is locally asymptotically stable if all the eigenvalues, $\lambda_{0 i}, i=1,2,3$ of the Jacobian matrix $J\left(E_{1}\right)$ satisfy the following condition $[1,2,3,10,12,13,17,18,24,25,26]$ :

$$
\left|\arg \left(\lambda_{1 i}\right)\right|>\frac{\alpha \pi}{2}
$$

The eigenvalues of the characteristic equation of $J\left(E_{1}\right)$ are $\lambda_{11}=-r, \lambda_{12}=d\left(R_{0}-1\right)$ and $\lambda_{13}=-\mu$. Hence $E_{0}$ is not locally asymptotically stable (since $E_{1}$ exists, it must be $R_{0}>1$; then $\lambda_{12}>0$ ).

We now discuss the asymptotic stability of a positive equilibrium point $E_{2}$ of the system given by (2.2). The Jacobian matrix $J\left(E_{2}\right)$ evaluated at a positive equilibrium is given as follows:

$$
J\left(E_{2}\right)=\left[\begin{array}{ccc}
-\frac{r X_{2}}{K}+\frac{b X_{2} Y_{2}}{\left(a+X_{2}\right)^{2}} & -\frac{b X_{2}}{a+X_{2}} & 0 \\
\frac{m a Y_{2}}{\left(a+X_{2}\right)^{2}} & 0 & -p Y_{2} \\
0 & 0 & -\mu+q Y_{2}
\end{array}\right]
$$


The characteristic equation of $J\left(E_{2}\right)$ as follows:

$$
\left(\lambda+\mu-q Y_{2}\right)\left(\lambda^{2}-B \lambda+C\right)=0
$$

where

$$
B=\frac{r d(a+K) X_{2}\left[(a+K) R_{0}-K\right]}{a m K^{2}} \text { and } C=\frac{a b m X_{2} Y_{2}}{\left(a+X_{2}\right)^{3}}>0
$$

For the characteristic equation (3.7), the roots are $\lambda_{21}=q Y_{2}-\mu$ and $\lambda_{22}, \lambda_{23}=\frac{1}{2}\left[B \pm \sqrt{B^{2}-4 C}\right]$.

Theorem 3.5. The equilibrium point $E_{2}$ of system (2.2) is locally asymptotically stable if and only if all the following conditions are satisfied:

i) $q Y_{2}-\mu<0$

ii) $R_{0}<\frac{K}{a+K}$ and

iii) $B<2 \sqrt{C} \cos \left(\frac{\alpha \pi}{2}\right)$.

Proof. It is clear that $\left|\arg \left(\lambda_{2 j}\right)\right|>\frac{\alpha \pi}{2}, j=2,3$, if and only if the conditions (ii) and (iii) hold.

Theorem 3.6. With respect to system (2.2), if $q Y_{2}-\mu<0$, the following statements can be obtained:

(a) If $R_{0} \leq \frac{K}{a+K}$, the equilibrium $E_{2}$ is locally asymptotically stable, for any $\alpha \in(0,1)$.

(b) If $0<B<2 \sqrt{C}$, the equilibrium $E_{2}$ is locally asymptotically stable if and only if $\alpha \in\left(0, \alpha^{*}\right)$, where $\alpha^{*}=\frac{2}{\pi}\left|\cos ^{-1}\left(\frac{B}{2 \sqrt{C}}\right)\right|$.

(c) If $B \geq 2 \sqrt{C}$, the equilibrium $E_{2}$ is unstable for any $\alpha \in(0,1)$.

Proof. The conclusions (a) and (c) are obvious. For the statement (b), due to $0<B<2 \sqrt{C}$, the equation (3.5) has two complex roots $\lambda_{22}, \lambda_{23}$, and their real part is $\frac{B}{2}>0$. Then $\left|\arg \left(\lambda_{2 j}\right)\right|=$ $\cos ^{-1}\left(\frac{B}{2 \sqrt{C}}\right), j=2,3$. Based on the condition $\cos ^{-1}\left(\frac{B}{2 \sqrt{C}}\right)=\frac{\alpha^{*} \pi}{2}, \alpha \in\left(0, \alpha^{*}\right)$ if and only if $\left|\arg \left(\lambda_{2 j}\right)\right|$ $>\frac{\alpha \pi}{2}, j=2,3$, it is concluded that Theorem 3.6 is true.

It can be concluded from the statements of Theorem 3.5 and Theorem 3.6 that the positive equilibrium is locally asymptotically stable if and only if $\alpha \in\left(0, \alpha^{*}\right)$. At $\alpha=\alpha^{*}$ a Hopf bifurcation is expected to occur. As increases above the critical value $\alpha^{*}$, the positive equilibrium is unstable, and a limit cycle is expected to appear in the proximity of $E_{2}$ due to the Hopf bifurcation phenomenon.

The analysis of periodic solutions in fractional dynamical systems is a new research topic that is generating great interest. Thus far, research indicates that exact periodic solutions in time invariant fractional systems are nonexistent. In terms of its application, the limit cycle detected in numerical simulations of a simple fractional neural network cannot represent an exact periodic solution of the system. Other research studies have also provided numerical evidence of limit cycles.

Remark 2. As of yet, there is no evidence of the existence of exact periodic solutions in autonomous fractional systems[29, 30]. However, numerical simulations have revealed limit cycles in many types of 
systems, including a fractional neural system[18], a fractional Van der Pol system[6], the fractional Chua and Chen systems[7], and a fractional financial system[4].

We now discuss the asymptotic stability of the positive equilibrium point $E_{3}$ of the system given by (2.2). The Jacobian matrix $J\left(E_{3}\right)$ is given as follows:

$$
J\left(E_{3}\right)=\left[\begin{array}{ccc}
-\frac{r X_{2}}{K}+\frac{b X_{3} Y_{3}}{\left(a+X_{3}\right)^{2}} & -\frac{b X_{3}}{a+X_{3}} & 0 \\
\frac{m a Y_{3}}{\left(a+X_{3}\right)^{2}} & 0 & -p Y_{3} \\
0 & q Z_{3} & 0
\end{array}\right] .
$$

Theorem 3.7. The interior equilibrium point $E_{3}$ is locally asymptotically stable.

Proof. The characteristic equation of $J\left(E_{3}\right)$ is as follows:

where

$$
\lambda^{3}+A \lambda^{2}+B \lambda+C=0
$$

$$
A=\frac{r R X_{2}}{K\left(a+X_{2}\right)}>0, B=\frac{a b m X_{2} Y_{2}}{\left(a+X_{2}\right)^{3}}+p q Y_{2} Z_{2} \text { and } C=\frac{r p q X_{2} Y_{2} Z_{2}}{K\left(a+X_{2}\right)}>0
$$

and

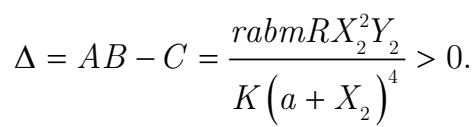

According to the Routh-Hurwitz criterion, we have $A>0, C>0$ and $\Delta>0$, therefore, $E_{2}$ is locally asymptotically stable.

Since $E_{3}$ is locally stable in the region $\Omega$, we will use Volterra-type Lyapunov functions (Lemma 3.1) to show in the following theorem[30].

Theorem 3.8. The interior equilibrium point $E_{3}$ is globally asymptotically stable.

Proof. A positive definite function $V(X, Y, Z)$ is defined as

$$
V(X, Y, Z)=M\left(X-X_{3}-X_{3} \ln \frac{X}{X_{3}}\right)+\left(Y-Y_{3}-Y_{3} \ln \frac{Y}{Y_{3}}\right)+N\left(Z-Z_{3}-Z_{3} \ln \frac{Z}{Z_{3}}\right),
$$

and positive constants $M, N$ will be chosen later. Then

$$
D_{t}^{\alpha} V(X, Y, Z)=M D_{t}^{\alpha}\left(X-X_{3}-X_{3} \ln \frac{X}{X_{3}}\right)+D_{t}^{\alpha}\left(Y-Y_{3}-Y_{3} \ln \frac{Y}{Y_{3}}\right)+N D_{t}^{\alpha}\left(Z-Z_{3}-Z_{3} \ln \frac{Z}{Z_{3}}\right) .
$$

By applying Lemma 3.1 [30] 


$$
\begin{aligned}
& D_{t}^{\alpha} V \leq M\left(1-\frac{X_{3}}{X}\right) D_{t}^{\alpha} X+\left(1-\frac{Y_{3}}{Y}\right) D_{t}^{\alpha} Y+N\left(1-\frac{Z_{3}}{Z}\right) D_{t}^{\alpha} Z \\
& =M\left(1-\frac{X_{3}}{X}\right)\left[r X\left(1-\frac{X}{K}\right)-\frac{b X Y}{a+X}\right]+\left(1-\frac{Y_{3}}{Y}\right)\left[-d Y+\frac{m X Y}{a+X}-p Y Z\right] \\
& +N\left(1-\frac{Z_{3}}{Z}\right)(-\mu Z+q Y Z) \\
& D_{t}^{\alpha} V \leq M\left(X-X_{3}\right)\left[r\left(1-\frac{X}{K}\right)-\frac{b Y}{a+X}\right]+\left(Y-Y_{3}\right)\left[-d+\frac{m X}{a+X}-p Z\right]+N\left(Z-Z_{3}\right)[-\mu+q Y] \\
& D_{t}^{\alpha} V \leq M\left(X-X_{3}\right)\left(\frac{r X_{3}}{K}+\frac{b Y_{3}}{a+X_{3}}-\frac{r X}{K}-\frac{b Y}{a+X}\right)+\left(Y-Y_{3}\right)\left(p Z_{3}-\frac{m X_{3}}{a+X_{3}}+\frac{m X}{a+X}-p Z\right) \\
& +N\left(Z-Z_{3}\right)\left[-q Y_{3}+q Y\right] \\
& =M\left(X-X_{3}\right)\left[-\frac{r}{K}\left(X-X_{3}\right)+\frac{b Y_{3}(a+X)-b Y\left(a+X_{3}\right)}{\left(a+X_{3}\right)(a+X)}\right] \\
& +\left(Y-Y_{3}\right)\left[-p\left(Z-Z_{3}\right)+\frac{m X\left(a+X_{3}\right)-m X_{3}(a+X)}{(a+X)\left(a+X_{3}\right)}\right]+q N\left(Z-Z_{3}\right)\left(Y-Y_{3}\right) \\
& D_{t}^{\alpha} V \leq M\left(X-X_{3}\right)\left[-\frac{r}{K}\left(X-X_{3}\right)+\frac{b Y_{3}\left(X-X_{3}\right)-a b\left(Y-Y_{3}\right)-b X_{3}\left(Y-Y_{3}\right)}{\left(a+X_{3}\right)(a+X)}\right] \\
& +\left(Y-Y_{3}\right)\left[-p\left(Z-Z_{3}\right)+\frac{a m\left(X-X_{3}\right)}{(a+X)\left(a+X_{3}\right)}\right]+q N\left(Z-Z_{3}\right)\left(Y-Y_{3}\right) \\
& =-M\left(X-X_{3}\right)^{2}\left[\frac{r}{K}-\frac{b Y_{3}}{\left(a+X_{3}\right)(a+X)}\right]+\left[\frac{a m-M\left(a b+b X_{3}\right)}{(a+X)\left(a+X_{3}\right)}\right]\left(X-X_{3}\right)\left(Y-Y_{3}\right) \\
& +(q N-p)\left(Z-Z_{3}\right)\left(Y-Y_{3}\right)
\end{aligned}
$$

If we take $M=\frac{a m}{a b+b X_{3}}>0, N=\frac{p}{q}$ and notice that when $E_{3}$ exists (and, consequently, is locally asymptotically stable), then

$$
\frac{r}{K}-\frac{b Y_{3}}{\left(a+X_{3}\right)(a+X)}>\frac{r X}{K(a+X)}>0 .
$$

Therefore, $D_{t}^{\alpha} V(X, Y, Z)$ is negative definite, and, consequently, $V$ is a Volterra-type Lyapunov function with respect to all solutions in the interior of the positive orthant, thereby proving the theorem.

\section{Numerical Methods and Simulations}

Because most fractional-order differential equations lack exact analytic solutions, approximation and numerical techniques must be used. There exist several different analytical and numerical methods for solving fractional-order differential equations. For numerical solutions of system (2.2), one can use the generalized Adams-Bashforth-Moulton method. To give the approximate solution by means of this algorithm, consider the following nonlinear fractional-order differential equation [10, 11, 14, 21]:

$$
\begin{gathered}
D_{t}^{\alpha} y(t)=f(t, y(t)), 0 \leq t \leq T \\
y^{k}(0)=y_{0}^{k}, k=0,1,2, \ldots, m-1 .
\end{gathered}
$$

This equation is equivalent to the Volterra integral equation: 


$$
y(t)=\sum_{k=0}^{m-1} y_{0}^{(k)} \frac{t^{k}}{k !}+\frac{1}{\Gamma(\alpha)} \int_{0}^{t}(t-s)^{\alpha-1} f(s, y(s)) d s
$$

Diethelm et al. used the predictor-corrector scheme [10, 11], based on the Adams-Bashforth-Moulton algorithm, to integrate Eq. (4.1). By applying this scheme to the fractional-order model for a tritrophic model consisting of tea plant, pest, and predator, and setting $h=\frac{T}{N}, t_{n}=n h, n=0,1,2, \ldots, N \in Z^{+}$one can discretize Eq. (4.1) as follows:

$$
\begin{aligned}
& X_{n+1}=X_{0}+\frac{h^{\alpha}}{\Gamma(\alpha+2)}\left[r X_{n+1}^{p}\left(1-\frac{X_{n+1}^{p}}{K}\right)-\frac{b X_{n+1}^{p} Y_{n+1}^{p}}{a+X_{n+1}^{p}}\right]+\frac{h^{\alpha}}{\Gamma(\alpha+2)} \sum_{j=1}^{n} a_{j, n+1}\left[r X_{j}\left(1-\frac{X_{j}}{K}\right)-\frac{b X_{j} Y_{j}}{a+X_{j}}\right] \\
& Y_{n+1}=Y_{0}+\frac{h^{\alpha}}{\Gamma(\alpha+2)}\left[-d Y_{n+1}^{p}+\frac{m X_{n+1}^{p} Y_{n+1}^{p}}{a+X_{n+1}^{p}}-p Y_{n+1}^{p} Z_{n+1}^{p}\right]+\frac{h^{\alpha}}{\Gamma(\alpha+2)} \sum_{j=1}^{n} a_{j, n+1}\left[-d Y_{j}+\frac{m X_{j} Y_{j}}{a+X_{j}}-p Y_{j} Z_{j}\right] \\
& Z_{n+1}=Z_{0}+\frac{h^{\alpha}}{\Gamma(\alpha+2)}\left[-\mu Z_{n+1}^{p}+q Y_{n+1}^{p} Z_{n+1}^{p}\right]+\frac{h^{\alpha}}{\Gamma(\alpha+2)} \sum_{j=1}^{n} a_{j, n+1}\left[-\mu Z_{j}+q Y_{j} Z_{j}\right]
\end{aligned}
$$

where

$$
\begin{aligned}
X_{n+1}^{p} & =X_{0}+\frac{1}{\Gamma(\alpha)} \sum_{j=0}^{n} b_{j, n+1}\left[r X_{j}\left(1-\frac{X_{j}}{K}\right)-\frac{b X_{j} Y_{j}}{a+X_{j}}\right] \\
Y_{n+1}^{p} & =Y_{0}+\frac{1}{\Gamma(\alpha)} \sum_{j=0}^{n} b_{j, n+1}\left[-d Y_{j}+\frac{m X_{j} Y_{j}}{a+X_{j}}-p Y_{j} Z_{j}\right] \\
Z_{n+1}^{p} & =Z_{0}+\frac{1}{\Gamma(\alpha)} \sum_{j=0}^{n} b_{j, n+1}\left[-\mu Z_{j}+q Y_{j} Z_{j}\right] \\
a_{j, n+1}= & \left\{\begin{array}{l}
(n-j-2)^{\alpha+1}+(n-j)^{\alpha+1}-2(n-j+1)^{\alpha+1}, 1 \leq j \leq n \\
1, j=0 \\
n^{\alpha-1}-(n-\alpha)(n+1), j=0
\end{array}\right. \\
b_{j, n+1}= & \frac{h^{\alpha}}{\alpha}\left[(n-j+1)^{\alpha}-(n-j)^{\alpha}\right], 0 \leq j \leq n .
\end{aligned}
$$

\section{Conclusion}

In this paper, we consider the fractional-order model for a tritrophic model consisting of tea plant, pest, and predator. We obtained a stability condition for equilibrium points, provided a numerical example, and verified our results. However, before drawing any conclusions, we must bear in mind that the equilibrium points are the same for both integer-order and fractional-order models, but the solution of the fractional-order model tends to the fixed point over a longer period of time. In addition, we must take into account that when dealing with real-life problems, one can determine the order of the system by using the collected data. Previous research has shown that the process of transforming a classical model into a fractional one is very sensitive to the order of differentiation $\alpha$; for instance, a small change in $\alpha$ may result in a substantial change in the final result. From the numerical, it is clear that the approximate solutions depend continuously on the fractional derivative $\alpha$. We used some documented data for several parameters, such as $r=1, K=2, a=0.9, b=1, d=1, m=3, p=1, \mu=1$ and $q=0.5$. The approximate solutions $X(t), Y(t)$ ? and $Z(t)$ are displayed in Figure 1 for the order of the fractional derivative $\alpha=0.95$. The results show that the concentrations of tea plants, pests, and predators all reach their equilibrium values as time passes. An important feature of the fractional-order model is that it controls the speed at which the solution to equilibrium is reached. It follows from Theorem 3.5, that $\alpha^{*}=0.985237$. From Theorem 3.6, it is known that when $\alpha<\alpha^{*}$, ? the trajectories 
converge to the equilibrium point, as shown in Figs. 1 and 2 ; whereas when $\alpha$ is increased to exceed $\alpha^{*}$, the origin loses its stability, and a Hopf-type bifurcation occurs, as shown in Fig. 3. Furthermore, Fig. 3 illustrates that the system (2.2) has a stable cycle trajectory.
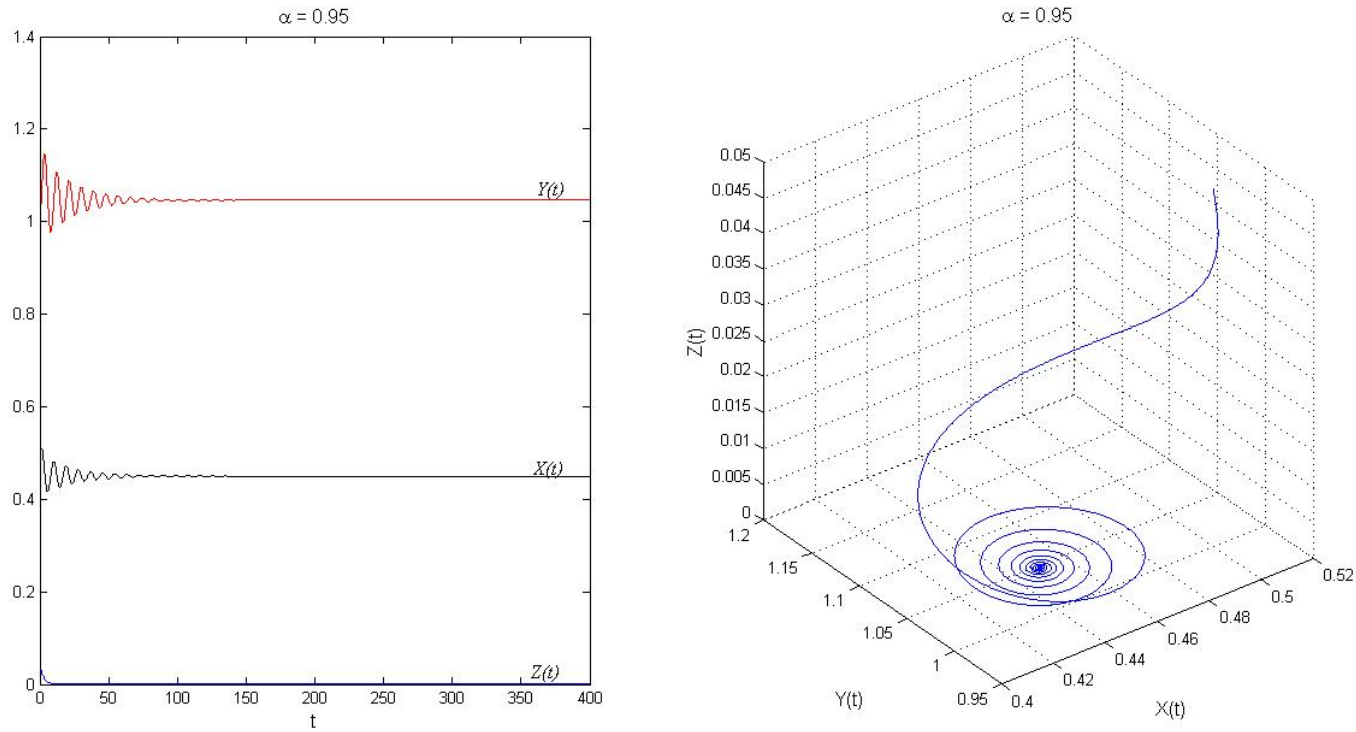

Figure 1. When $\alpha=0.95$ the trajectory of system (2.2) converges to the equilibrium $E_{2}$.
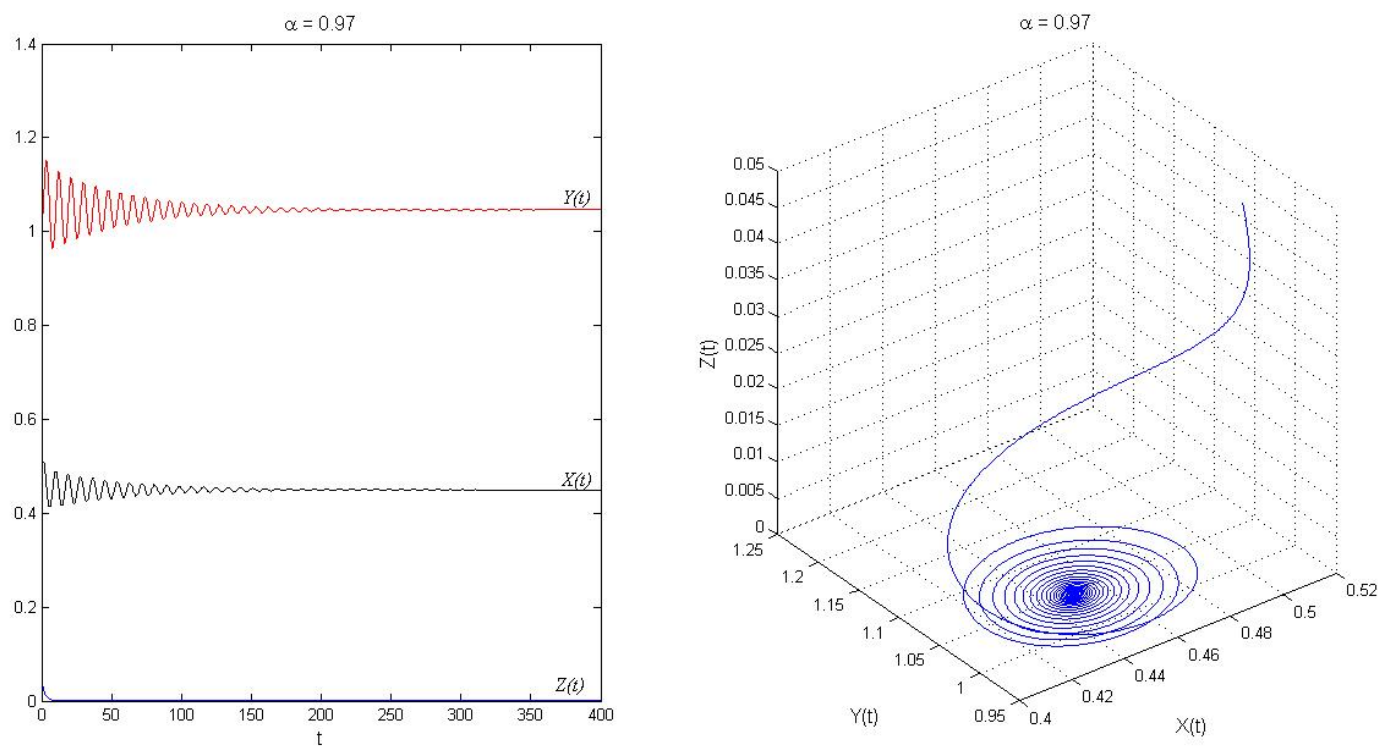

Figure 2. When $\alpha=0.97$ the trajectory of system (2.2) converges to the equilibrium $E_{2}$. 

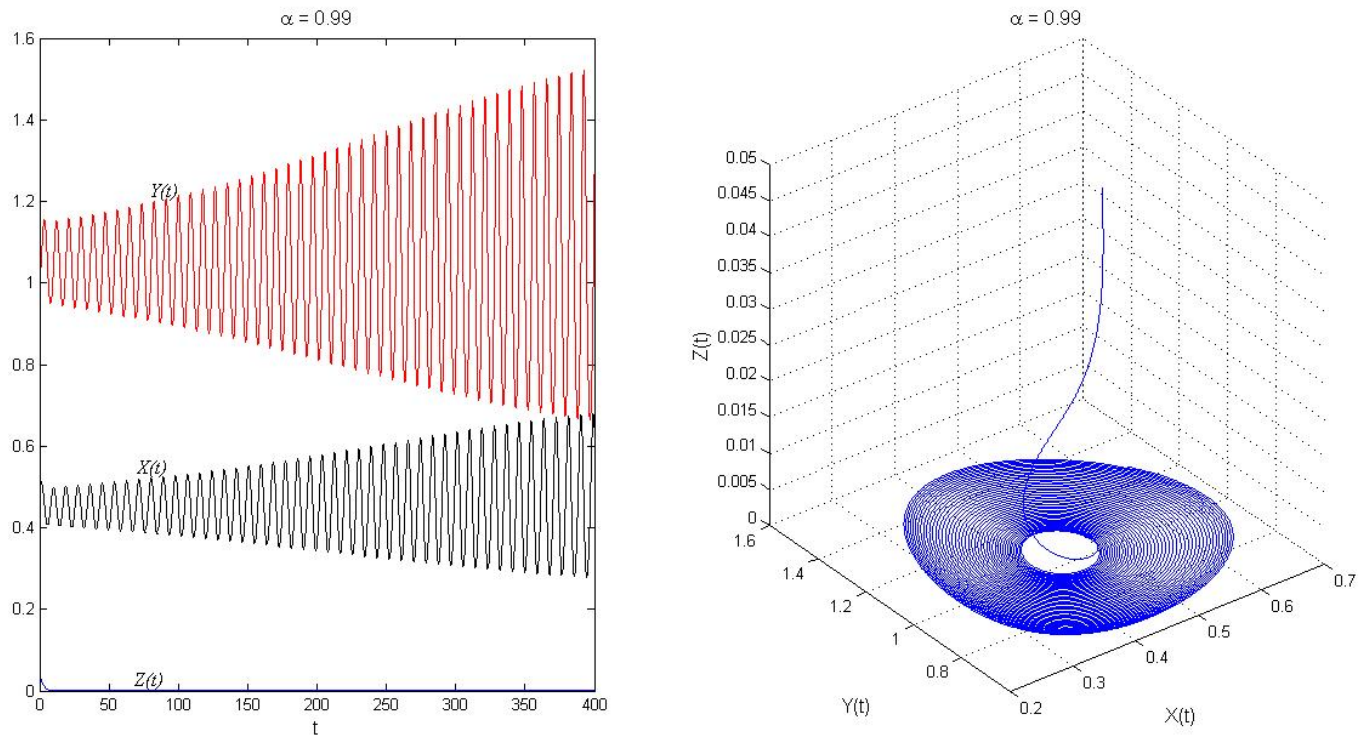

Figure 3. When $\alpha=0.99$ the trajectory of system (2.2) converges to an asymptotically stable limit cycle.

\section{References}

1. E. Ahmed, A. M. A. El-Sayed, E. M. El-Mesiry and H. A. A. El-Saka; Numerical solution for the fractional replicator equation, IJMPC, 16 (2005), 1.9.

2. E. Ahmed, A. M. A. El-Sayed, H. A. A. El-Saka; On some Routh-Hurwitz conditions for fractional order differential equations and their applications in Lorenz, Rossler, Chua and Chen systems, Physics Letters A, 358 (2006), 1.4.

3. E. Ahmed, A. M. A. El-Sayed, H. A. A. El-Saka; Equilibrium points, stability and numerical solutions of fractional-order predator-prey and rabies models, J. Math. Anal. Appl, 325 (2007), 542.553.

4. M. S. Abd-Elouahab, N. E. Hamri, and J. Wang, Chaos control of a fractional-order financial system, Mathematical Problems in Engineering, 2010 (2010), 18 pages.

5. R. M. Anderson; R. M. May; Infectious Diseases of Humans: Dynamics and Control, Oxford University Press, Oxford, 1991.

6. R. S. Barbosa, J. A. T. MacHado, B. M. Vinagre, and A. J. Calderón, Analysis of the van der Pol oscillator containing derivatives of fractional order, Journal of Vibration and Control, 13 (2007), 1291.1301.

7. D. Cafagna and G. Grassi, Fractional-order Chua.s circuit: time-domain analysis, bifurcation, chaotic behavior and test for chaos, International Journal of Bifurcation and Chaos, 18 (2008), 615.639.

8. S. C. Das and K. C. Bar Ua, Scope of bio-control of pests and diseases in tea plantations, Tea Research Association, Tocklai Experimental Station, Jorhat- 785008, Assam.

9. L. Debnath; Recent applications of fractional calculus to science and engineering, International Journal of Mathematics and Mathematical Sciences, 54 (2003), 3413. 3442.

10. K. Diethelm, N. J. Ford; Analysis of fractional differential equations, J Math Anal Appl, 256 (2002), 229.248.

11. K. Diethelm, N. J. Ford, A.D. Freed; A predictor-corrector approach for the numerical solution of fractional differential equations, Nonlinear Dyn, 29 (2002), 3.22.

12. M. Elshahed and A. Alsaedi; The Fractional SIRC model and Influenza A, Mathematical Problems in Engineering, Article ID 480378 (2011), 1.9.

13. M. Elshahed, F. Abd El-Naby, Fractional calculus model for childhood diseases and vaccine Applied Mathematical Sciences, 8 (2014), 4859 - 4866.

14. R. Garrappa, Trapezoidal methods for fractional differential equations: Theoretical and computational aspects, Mathematics and Computers in Simulation, 110 (2015), 96.112.

15. B. Han, Chen. Z., Behavioral and electrophysiological responses of natural enemies to synonymous from tea shoots and kairomones from tea aphids, Toxoptera aurantii, J Chem Ecol. 28 (2002), 2203-19. 
16. H. Jonathan Crane and Carlos F. Balerdi, Tea Growing in the Florida Home Land-scape, University of Florida. Original publication, 2005.

17. E. Kaslik and S. Sivasundaram, Nonlinear dynamics and chaos in fractional-order neural networks, Neural Networks, 32 (2012), 245.256.

18. E. Kaslik and S. Sivasundaram, Non-existence of periodic solutions in fractional-order dynamical systems and a remarkable difference between integer and fractional-order derivatives of periodic functions, Nonlinear Analysis: Real World Applications, 13 (2012), 1489.1497 .

19. A. A. Kilbas.; H. M. Srivastava.; and J. J. Trujillo.; Theory and Applications of Fractional Differential Equations, Elsevier Science, Amsterdam, The Netherlands, 204 (2006).

20. S. Kyu Choi, B. Kang, and N. Koo, Stability for Caputo Fractional Differential Systems, Hindawi Publishing Corporation, Abstract and Applied Analysis, 2014 (2014), 6 pages.

21. C. Li, C. Tao; On the Fractional Adams method, Computers and Mathematics with Applications, 58 (2009), 1573.1588 .

22. A. Maiti, A. K. Pal and G. P. Samanta, Usefulness of biocontrol of pests in tea: a mathematical model, Math. Model. Nat. Phenom. 3 (2008), 96-113.

23. D. Matignon; Stability results for fractional differential equations with applications to control processing, Computational Engineering in Systems and Applications, Multi-conference, 2 (1996), 963-968.

24. A. E. Matouk, A. A. Elsadany, E. Ahmed, H. N. Agiza, Dynamical behavior of fractional-order Hastings.Powell food chain model and its discretization, Communications in Nonlinear Science and Numerical Simulation 27 (2015), 153-167.

25. A. E. Matouk, A. A. Elsadany, Dynamical behaviors of fractional-order Lotka. Volterra predator-prey model and its discretization, Journal of Applied Mathematics and Computing 49 (2015), 269-283.

26. A. E. Matouk, A. A. Elsadany, Dynamical analysis, stabilization and discretization of a chaotic fractional-order GLV model, http://link.springer.com/article/10.1007/s11071-016-2781-6., 2015

27. M.S.A. Mamun and M. Ahmed, Prospect of indigenous plant extracts in tea pest management, Int. J. Agril. Res. Innov. \& Tech. 1, 1\&2 (2011): 16-23,

28. I. Podlubny, Fractional Differential Equations, Academic Press, New York, NY, USA (1999).

29. M. S. Tavazoei and M. Haeri, A proof for nonexistence of periodic solutions in time invariant fractional order systems, Automatica, 45 (2009), 1886.1890.

30. C. Vargas-De-León, Volterra-type Lyapunov functions for fractional-order epidemic systems, Commun Nonlinear Sci Numer Simulat, 24 (2015), 75.85

31. http://www.tocklai.net/list-of-manufacturers-registered-for-production-and-sale-of-bio-pesticides/ 